\title{
Colonic diverticulosis is not a risk factor for colonic adenoma
}

This article was published in the following Dove Press journal:

Therapeutics and Clinical Risk Management

\author{
Wandong Hong, ${ }^{1,2, *}$ Lemei \\ Dong, ${ }^{1, *}$ Maddalena Zippi, ${ }^{3, *}$ \\ Simon Stock, ${ }^{4}$ Wujun \\ Geng, ${ }^{5, *}$ Chunfang Xu, ${ }^{2}$ \\ Mengtao Zhou $^{6}$ \\ 'Department of Gastroenterology \\ and Hepatology, The First Affiliated \\ Hospital, Wenzhou Medical \\ University, Wenzhou, Zhejiang, China; \\ ${ }^{2}$ Department of Gastroenterology, \\ The First Affiliated Hospital, Soochow \\ University, Suzhou, Jiangsu, China; \\ ${ }^{3}$ Unit of Gastroenterology and \\ Digestive Endoscopy, Sandro Pertini \\ Hospital, Rome, Italy; ${ }^{4}$ Department \\ of Surgery, World Mate Emergency \\ Hospital, Battambang, Cambodia; \\ ${ }^{5}$ Department of Anesthesiology, The \\ First Affiliated Hospital, Wenzhou \\ Medical University, Wenzhou, Zhejiang, \\ China; ${ }^{6}$ Department of Surgery, The \\ First Affiliated Hospital, Wenzhou \\ Medical University, Wenzhou, \\ Zhejiang, China \\ *These authors contributed equally \\ to this work
}

Background and aims: Colonic diverticulosis may represent a risk factor for colonic adenomas by virtue of the fact that evolving data suggest that these 2 conditions may share common risk factors such as Western dietary pattern and physical inactivity. This study aims to investigate the association between colonic diverticulosis and colonic adenomas in mainland China.

Methods: We conducted a cross-sectional study on patients who underwent colonoscopic examination between October 2013 and December 2014 in a university hospital in mainland China. Age, gender, colonic adenomas, advanced adenomas, and distribution of diverticulosis were recorded during the procedures. Multivariate logistic regression and stratified analysis were used to evaluate the associations between the prevalence of diverticulosis and age, sex, and presence of colonic adenomas and advanced adenomas.

Results: A total of 17,456 subjects were enrolled. The prevalence of colonic diverticulosis and adenoma was $2.4 \%$ and $13.2 \%$, respectively. With regard to distribution of diverticula, most $(365 / 424,86.1 \%)$ were right-sided. Multiple logistic regression analysis suggested that age and male gender were independent risk factors for adenoma and advanced adenoma. There was no relationship between diverticulosis or location of diverticulosis and presence of adenoma and advanced adenoma adjusting by age and gender. In a stratified analysis according to age and gender, similar results were also noted.

Conclusion: There was no statistical relationship between diverticulosis and the risk of adenoma and advanced adenoma. Our results may not be generalized to the Western population due to the fact that left-sided diverticular cases were very small in our study.

Keywords: colonic adenoma, advanced adenoma, colonic diverticulosis, prevalence, risk factor

\section{Introduction}

Evolving data suggested that colonic diverticular disease and colonic adenomas may share common risk factors such as Western dietary pattern, ${ }^{1}$ lifestyle factors (overweight, obesity, and physical inactivity), ${ }^{2}$ and cigarette smoking. ${ }^{3}$ Both the prevalence of diverticulosis and colonic adenomas increased with age, ${ }^{4,5}$ and varied with geography and ethnics. ${ }^{4,5}$ The proposed common mechanisms for diverticulosis and colonic adenoma formation include lack of dietary fiber, increased saturated fats, and slow colonic transit time. ${ }^{6}$ Therefore, there is a hypothesis that diverticulosis is a risk factor for adenomas. However, the relationship between the presence of diverticulosis and adenomas is still controversial in the literature. Most studies suggest that diverticulosis is a risk factor for colonic adenomas while no correlation between diverticulosis and adenomas was noted in other studies. ${ }^{6-12}$ In addition, most of these studies were performed in industrialized countries in the West, where there is a high prevalence of colonic diverticulosis varying from $20.3 \%$ to $42 \% .{ }^{12,13}$ The anatomic distribution pattern 
of diverticulosis is predominantly left-sided in the Western countries and right-sided in Asia. ${ }^{3,4,14}$ The pathophysiology of right-sided diverticulosis remains unclear. ${ }^{15}$ It is thought that the majority of right-sided diverticulosis might be congenital and self-limiting., ${ }^{3,16,17}$ Left-sided diverticulosis is thought to be acquired as a result of low-fiber diet and changes in colonic motility and the connective tissue of the colonic wall. ${ }^{18}$ Our recent study showed that Chinese people have a low prevalence (1.97\%) of diverticulosis and most diverticulosis are right-sided $(85.3 \%)$ in the region of mainland China. ${ }^{4}$ As to colonic adenomas, it was estimated that $30 \%$ of Western populations have colonic adenomas while a lower rate $(10 \%-15 \%)$ is noted in Asia and Africa. ${ }^{19}$ Therefore, there is a question about whether the presence of diverticulosis is associated with colonic adenomas in an Asian population with a low prevalence of diverticulosis and adenomas such as mainland China. ${ }^{4}$

For the aforementioned reasons, this study aims to investigate the association between colonic diverticulosis and colonic adenomas in mainland China.

\section{Materials and methods Inclusion and exclusion criteria}

Patients who underwent colonoscopy examination in the First Affiliated Hospital, Wenzhou Medical University, between October 2013 and December 2014 were eligible. As described in our previous study, ${ }^{4}$ exclusion criteria included as follows: therapeutic colonoscopy, prior colonic resection, colon cancer, incomplete inadequate bowel preparation or colonoscopy examination, and repeat colonoscopy examination within 1 year (for which only the most recent colonoscopy examination was included in our study).

\section{Ethics}

This study protocol was approved by the Ethics Committee of the First Affiliated Hospital of Wenzhou Medical University. This study was performed according to the principles expressed in the Declaration of Helsinki. Written informed consent, for the use of their data for future research purposes, was obtained from the subjects.

\section{Data collection and definition}

The definition of complete colonoscopy and classification of bowel preparation was described by Ashktorab et al. ${ }^{20}$ Diverticulosis was defined as the presence of 1 or more colonic diverticula irrespective of whether they are symptomatic or complicated. ${ }^{4}$ Age, gender, and distribution of diverticulosis were recorded. Age was parsed into a categorical variable with 4 groups, $\leq 40,41-50,51-60$, and $>60$ years old. Colonic diverticulosis was divided by location into left (descending colon, sigmoid colon, and rectum), right (cecum, ascending colon, and transverse colon), and bilateral (right, transverse, and left sections of the colon). ${ }^{4}$ Adenomas were defined as 1 or more colorectal adenomas present on colonoscopy irrespective of size and location. ${ }^{12}$ Hyperplastic polyps were not considered. ${ }^{6}$ Advanced adenomas were defined as adenomas $>1 \mathrm{~cm}$ or with villoglandular histology or highgrade dysplasia. ${ }^{12}$

\section{Statistical analysis}

Categorical values were described by count and proportions and compared by the $\chi^{2}$ test. Continuous values were expressed as mean \pm SD and compared using the independent samples $t$-test. Linear trend of categorical variables was tested by a Royston extension of the Cochran-Armitage test. A multivariate logistic regression analysis was used to evaluate the associations between the prevalence of diverticulosis and patient age category, sex, and presence of colonic adenomas. Odds ratios (OR) were calculated with 95\% CI. Two-sided $P$-values below 0.05 were considered significant.

\section{Results \\ Baseline characteristics and effects of gender and age}

As showed in Figure 1, a total of 26,352 colonoscopy examinations were performed between October 2013 and December 2014 in our hospital. A total of 17,456 (58.1\% males) subjects were enrolled in this study. Overall, 424 subjects had colonic diverticulosis during the examination, a prevalence of $2.4 \%$. Patients with diverticula were more likely to be older (mean age: 53.5 vs $49, P<0.001$ ) and male (75.2\% vs $57.7 \%, P<0.001)$ than those without diverticula (Figure 1). With regard to distribution of diverticula, most (365/424, 86.1\%) were right-sided.

Among the 17,456 participants, 2,307 (13.2\%) had adenoma and 596 (3.4\%) had advanced adenoma on colonoscopy. Of the patients presenting with advanced adenoma, 491 (82.9\%) had size $>1 \mathrm{~cm}, 242(40.6 \%)$ had villous component, and 99 (16.6\%) had high-grade dysplasia (Figure 1). Patients with adenoma were more likely to be older (mean age: 56.1 vs $50.0, P<0.001$ ) and male $(67.9 \%$ vs $56.6 \%, P<0.001)$ than those without adenoma (Table 1). Furthermore, the prevalence of adenoma was $4.8 \%, 10.1 \%, 17.3 \%$, and $24.5 \%$ at age groups $\leq 40,41-50$, $51-60$, and $>60$ years, respectively. This finding indicates a greater increase in age was associated with an increased 


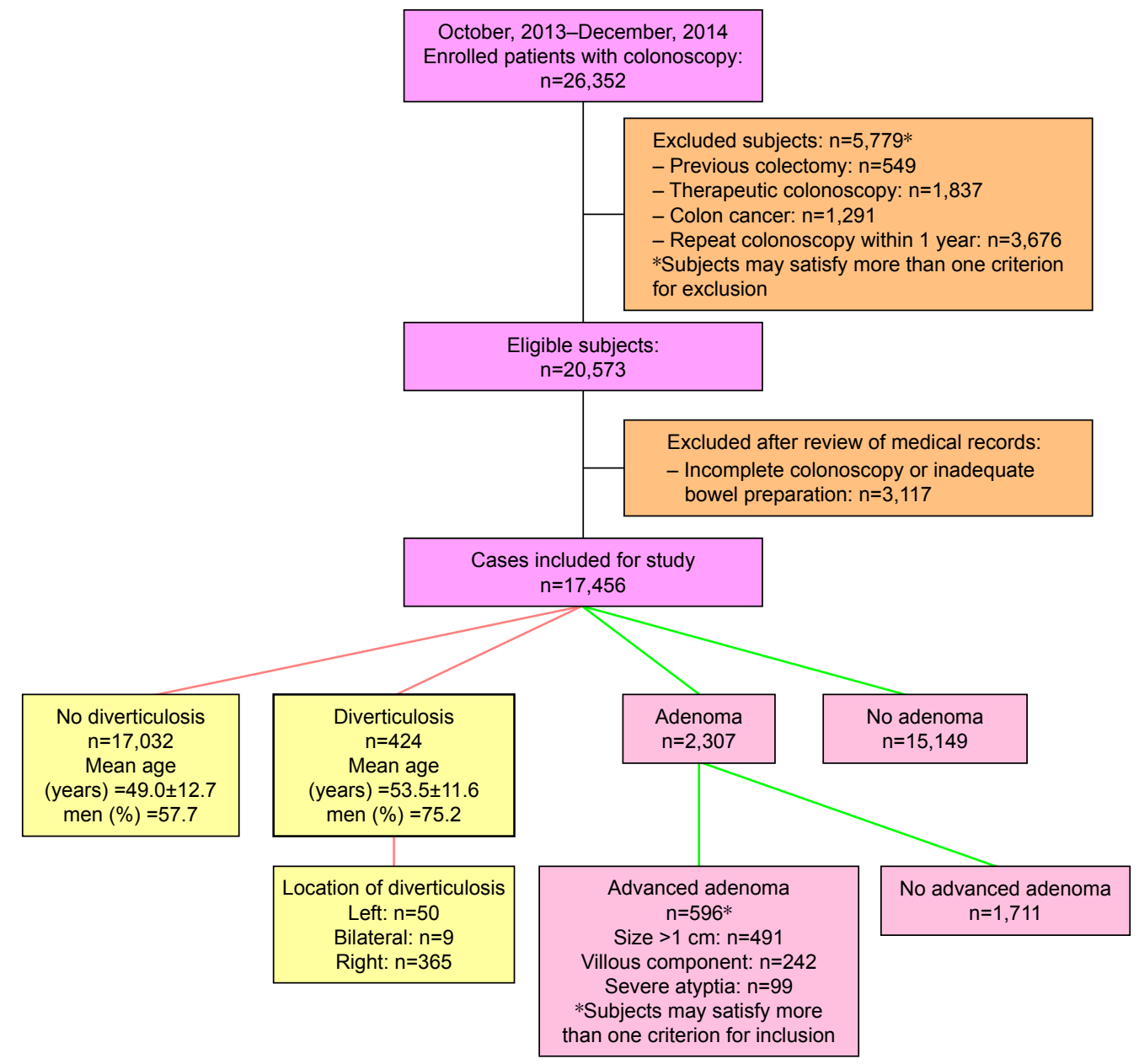

Figure I Flow diagram of patients included in the study and baseline characteristics.

adenoma detection rate (Test for trend: $P<0.001$ ) (Table 1). Multivariate logistic regression analysis indicated that male gender (OR 1.69; 95\% CI, 1.54-1.86; $P<0.001$ ) and age were independently associated with presence of adenoma (Figure 2). Using patients in age group $\leq 40$ as reference, the

Table I Comparison of gender, age, and prevalence of diverticulosis between patients with or without adenoma $(\mathrm{N}=17,456)$

\begin{tabular}{llll}
\hline Variable & Adenoma & No adenoma & $P$-value \\
\hline Gender, $\mathrm{n}$ & & & $<0.00 \mathrm{I}$ \\
$\quad$ Women & $74 \mathrm{I}(32.1 \%)$ & $6,57 \mathrm{I}(43.4 \%)$ & \\
$\quad$ Men & $\mathrm{I}, 566(67.9 \%)$ & $8,579(56.6 \%)$ & \\
Age, years (mean \pm SD) & $56.1 \pm \mathrm{II} .8$ & $50.0 \pm 12.5$ & $<0.00 \mathrm{I}$ \\
Age group, $\mathrm{n}$ & & & $<0.00 \mathrm{I}$ \\
$\quad \leq 40$ & $222(4.8 \%)$ & $4,385(95.2 \%)$ & \\
$4 \mathrm{I}-50$ & $537(10.1 \%)$ & $4,788(89.9 \%)$ & \\
$5 \mathrm{I}-60$ & $708(17.3 \%)$ & $3,393(82.7 \%)$ & \\
$>60$ & $840(24.5 \%)$ & $2,583(75.5 \%)$ & \\
Diverticulosis & & & 0.004 \\
$\quad$ Yes & $76(3.3 \%)$ & $348(2.3 \%)$ & \\
No & $2,23 \mathrm{I}(96.7 \%)$ & $14,80 \mathrm{I}(97.7 \%)$ & \\
\hline
\end{tabular}

OR of the presence of adenoma for patients with age groups 41-50, 51-60, and $>60$ years were 2.26 (95\% CI, 1.92-2.65; $P<0.001), 4.23$ (95\% CI, 3.61-4.95; $P<0.001)$, and 6.57 (95\% CI, 5.62-7.68; $P<0.001$ ), respectively (Figure 2).

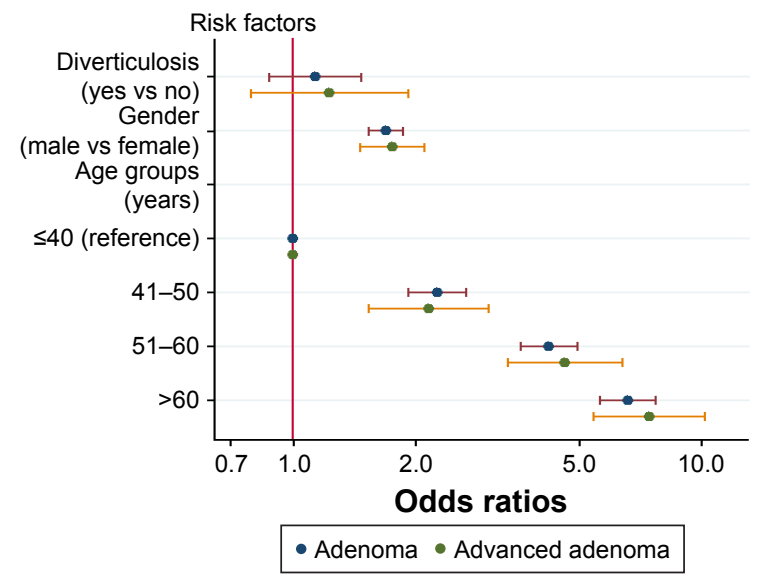

Figure 2 Logistic regression plot of odds ratios and $95 \% \mathrm{Cls}$. Note: Risk factors for adenoma and advanced adenoma. 


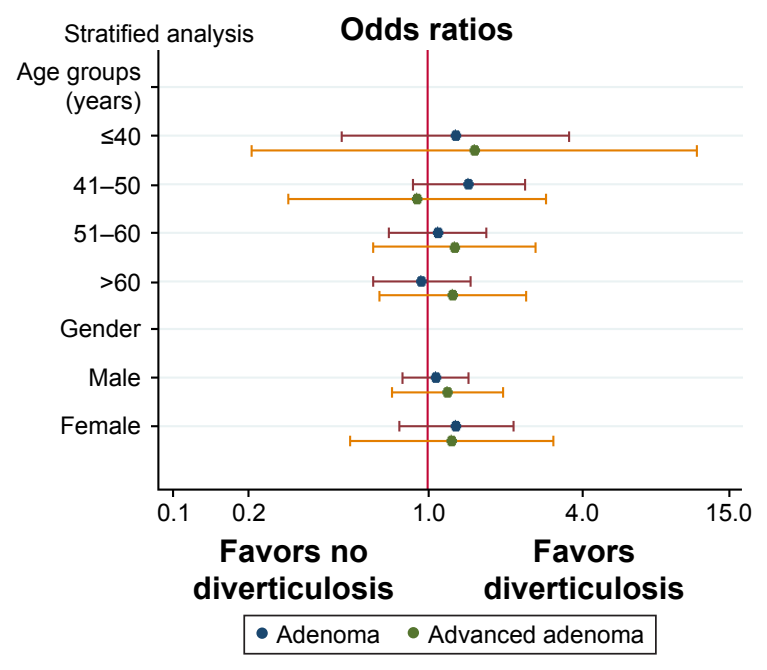

Figure 3 Logistic regression plot of odds ratios and $95 \% \mathrm{Cls}$.

Note: Subgroup analysis of relationship between diverticulosis and adenoma, and advanced adenoma in patients of different age groups and gender.

\section{Association of diverticulosis with adenoma and advanced adenoma}

At univariable analysis, prevalence of diverticulosis was significantly higher in patient with adenoma than in those without adenoma (3.3\% vs $2.3 \%, P=0.004)$ (Table 1$)$. Multivariate logistic regression analysis indicated that diverticulosis was not associated with an increased risk of adenomas (OR 1.14; 95\% CI, 0.88-1.47; $P=0.324$ ) or advanced adenomas (OR 1.23; 95\% CI, 0.79-1.91; $P=0.358$ ) after adjustment for gender and age (Figure 2).

Stratified analysis was performed according to different age groups and gender. As shown in Figure 3, the presence of diverticulosis was not associated with an increased risk of adenoma or advanced adenoma in every age group after adjustment by gender. Similarly, no statistical relationship between diverticulosis and adenoma or advanced adenoma was found in either male or female groups after adjustment by age.

\section{Distribution of diverticula, adenoma, and advanced adenoma}

At univariable logistic analysis, the presence of diverticulosis in the right side of the colon was associated with increased adenoma detection (OR 1.46; 95\% CI, 1.12-1.92; $P=0.006$ ) but not advanced adenoma (OR 1.48; 95\% CI, 0.92-2.40; $P=0.109$ ) (Figure 4A). Multivariable logistic regression analysis indicated that right-sided diverticulosis was not associated with an increased risk of adenomas (OR 1.21; 95\% CI, 0.91-1.59; $P=0.185)$ or advanced adenomas (OR 1.22; 95\% CI, 0.75-1.99; $P=0.418$ ) after adjustment for gender and age (Figure 4B). Both univariable and multivariable logistic analysis showed that the presence of diverticulosis in left or both sides of the colon was not associated with an increased adenoma or advanced adenoma detection rate (Figure 4).

Because most of our patients had right-sided diverticulosis, stratified analysis was performed in these patients according to different age groups and gender. As shown in Figure 5, the presence of diverticulosis in the right side of the colon was not associated with an increased risk of adenoma or advanced adenoma in any age group after adjustment by gender. Similarly, no statistical relationship between rightsided diverticulosis and adenoma or advanced adenoma was found in either male or female groups after adjustment by age.
A

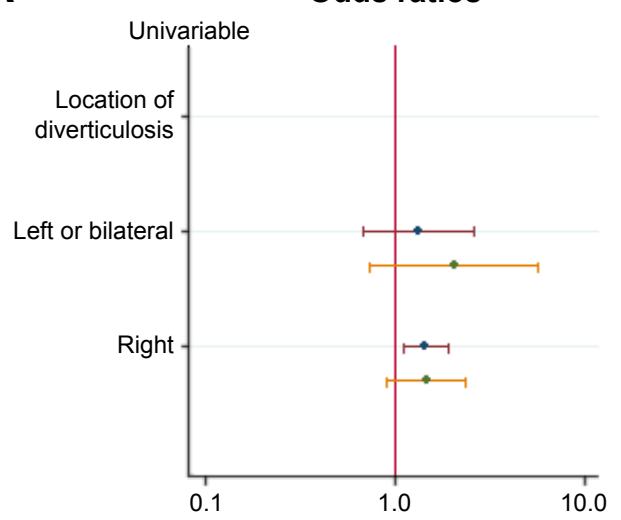

B

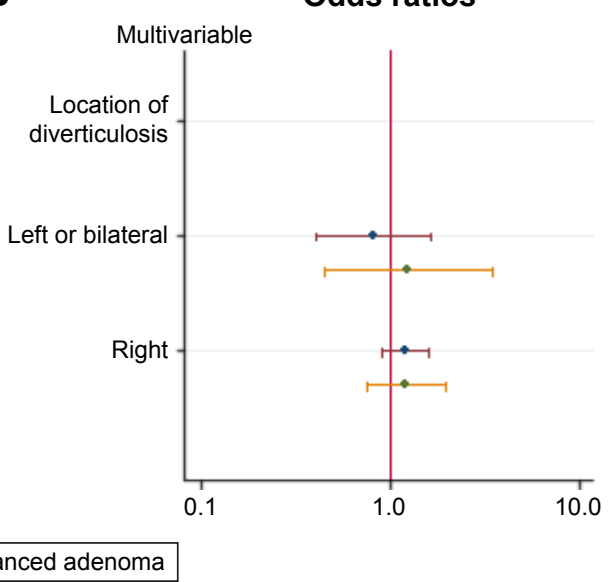

Figure 4 Logistic regression plot of odds ratios and $95 \% \mathrm{Cls}$.

Notes: Relationship between location of diverticula in colon, adenoma and advanced adenoma: (A) univariable logistic regression analysis; (B) multivariable logistic regression analysis adjusted by gender and age. 


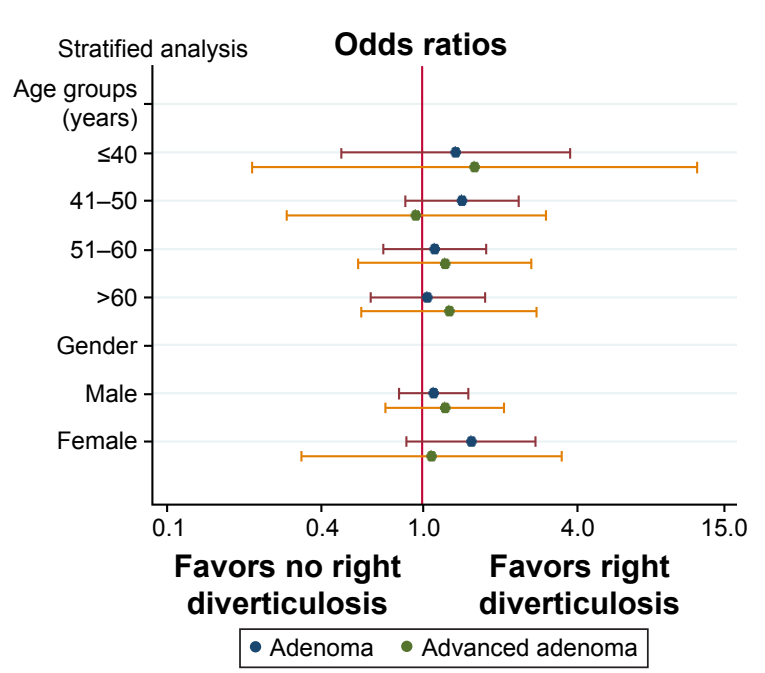

Figure 5 Logistic regression plot of odds ratios and $95 \% \mathrm{Cls}$

Note: Subgroup analysis of relationship between right side diverticulosis and adenoma, and advanced adenoma in patients with different age groups and gender.

\section{Discussion}

The outcomes of the present study prove that: 1) age and male gender but not colonic diverticulosis were associated with increased risk of adenoma and advanced adenoma; 2) no statistical relationship was found between the distribution of diverticula and the presence of adenoma and advanced adenoma.

The prevalence of diverticulosis varies worldwide in different populations. The overall prevalence of diverticulosis in this study was $2.4 \%$, which is similar to our previous report, ${ }^{4}$ but it was significantly lower than the recent results from Singapore (45.0\%) and Japan (20.0\%-25.8\%).,13,21,22 This difference may be mainly attributed to different lifestyle, dietary habits, ${ }^{23}$ race, genetic predisposition, and the proportion of elderly people who underwent colonoscopy examination since diverticulosis is age-dependent. ${ }^{4,24}$

Patients with diverticula were more likely to be older and male than those without diverticula (Figure 1), which was consistent with the study by Nagata et al. ${ }^{13}$ With regard to distribution of diverticula, $86.1 \%$ cases of diverticulosis in our data were located in right side of the colon. This is consistent with the previous observation that the anatomic distribution pattern of diverticulosis is predominantly leftsided in the West and right-sided in Asia.

Our univariable analysis suggested that an increase in age was proportionally associated with an increased adenoma detection rate (Test for trend: $P<0.001$ ) (Table 1). Multivariate logistic regression analysis indicated that male gender and age were independent risk factors for both adenoma and advanced adenoma (Figure 2). Corley et al similarly showed that the prevalence of detected adenoma increases substantially with age and is much higher in men. ${ }^{5}$ BurnettHartman et al suggested that there was a strong association between old age and advanced adenomas. ${ }^{25}$ Tao et al suggested that age and male sex were associated significantly with the risk of advanced colonic neoplasms. ${ }^{26}$ The gender effects on pathogenesis of diverticulosis and adenoma are unclear though it is now being widely recognized that there are important sex differences in many diseases. ${ }^{27}$ A growing body of evidence shows there are some sex-associated differences in gut bacterial flora composition and gut luminal metabolic activity. ${ }^{28}$

Data on the association between diverticulosis and the presence of colonic adenoma and advanced adenoma is somewhat conflicting. Most studies found that there was a positive association between diverticulosis and the colonic adenoma detection rate. ${ }^{6-10}$ However, these studies have some limitations such as small sample, ${ }^{8,10}$ inclusion of patients with hyperplastic polyps and adenocarcinoma, or only investigating patients with a positive fecal immunochemical test. ${ }^{6,7,9}$ In addition, stratified analysis according to age and gender was infrequently performed. Our study suggested that, at univariable analysis, the prevalence of diverticulosis was significantly higher in patients with adenoma than those without adenoma (3.3\% vs 2.3\%) (Table 1). Multivariate logistic regression analysis indicated that diverticulosis was not associated with increased adenoma and advanced adenoma detection after adjustment by age and gender (Figure 2). Furthermore, at stratified analysis according to age and gender (Figure 3), the presence of diverticulosis was not associated with an increased risk of adenoma or advanced adenoma in any age group after adjustment by gender. Similarly, no statistical relationship between diverticulosis and adenoma or advanced adenoma was found in either male or female groups after adjustment by age. These results are consistent with suggestions from a prospective study and a retrospective study with 4,241 colonoscopies that diverticulosis was not a risk factor for adenoma or advanced adenoma. ${ }^{11,12}$ We also did not find any association between distribution of diverticulosis and adenoma and advanced adenoma at multivariable logistic regression when adjusted by age and gender (Figure 4). Because most of our patients had rightsided diverticulosis, stratified analysis was performed in these patients according to different age groups and gender. As shown in Figure 5, the presence of diverticulosis in the right side of the colon was not associated with an increased risk of adenoma or advanced adenoma in any age group after adjustment by gender. Similarly, no statistical relationship 
between diverticulosis in the right side of the colon and adenoma or advanced adenoma was found in either male or female groups after adjustment by age.

The strengths of this study include a large sample size that gives the study enough statistical power to detect an association between diverticulosis and adenoma. To the best of our knowledge, this is the first study to investigate relationship between diverticulosis and presence of adenoma and advanced adenoma in Chinese population stratified by age and gender in mainland China. There are, however, some limitations in the present study. First, this is a retrospective cross-sectional study, precluding the assessment of other risk factor such as dietary pattern, cigarette smoking, and alcohol consumption. Second, our results were from a single center in a medium-sized city of China, and lifestyle and dietary habits vary in different regions in China. ${ }^{29}$ Therefore, our results may not be generalized to the entire Chinese population in mainland China. On the other hand, our results may also not be generalized to Western population due to the fact that left-sided diverticular cases were very small in our study. At last, similar to previous studies, ${ }^{6,7,9,11}$ we included colonoscopies for different indications (including screening and symptomatic patients) rather than using data from screening colonoscopies exclusively, which may have had the effect of increasing the adenoma detection rates. Though utilizing a mixed cohort of consecutive patients might reflect better about what is encountered in general practice, our hospital-based study results might not be generalized for healthy population.

In summary, age and male gender were associated with increased risk of adenoma and advanced adenoma; but no statistical relationship was found between diverticula and the risk of adenoma and advanced adenoma.

\section{Author contributions}

All authors contributed toward data analysis, drafting and revising the paper and agree to be accountable for all aspects of the work.

\section{Disclosure}

The authors report no conflicts of interest in this work.

\section{References}

1. Makambi KH, Agurs-Collins T, Bright-Gbebry M, Rosenberg L, Palmer JR, Adams-Campbell LL. Dietary patterns and the risk of colorectal adenomas: the Black Women's Health Study. Cancer Epidemiol Biomarkers Prev. 2011;20(5):818-825.

2. Fu Z, Shrubsole MJ, Smalley WE, et al. Lifestyle factors and their combined impact on the risk of colorectal polyps. Am J Epidemiol. 2012;176(9):766-776.
3. Nagata N, Niikura R, Shimbo T, et al. Alcohol and smoking affect risk of uncomplicated colonic diverticulosis in Japan. PLoS One. 2013;8(12):e81137.

4. Hong W, Geng W, Wang C, et al. Prevalence of colonic diverticulosis in mainland China from 2004 to 2014. Sci Rep. 2016;6:26237.

5. Corley DA, Jensen CD, Marks AR, et al. Variation of adenoma prevalence by age, sex, race, and colon location in a large population: implications for screening and quality programs. Clin Gastroenterol Hepatol. 2013;11(2):172-180.

6. Muhammad A, Lamendola O, Daas A, Kumar A, Vidyarthi G. Association between colonic diverticulosis and prevalence of colorectal polyps. Int J Colorectal Dis. 2014;29(8):947-951.

7. Rondagh EJ, Sanduleanu S, le Clercq CM, Winkens B, Masclee AA. Diverticulosis and colorectal polyps at younger age: a possible link? Eur J Gastroenterol Hepatol. 2011;23(11):1050-1055.

8. Gohil VB, Patrie JT, Shami VM, et al. Colonic diverticulosis is associated with an increased adenoma detection rate in patients undergoing first-time screening colonoscopy. J Interv Gastroenterol. 2012; 2(2):70-75.

9. Morini S, Ridola L, Hassan C, et al. Association between diverticulosis and colonic neoplastic lesions in individuals with a positive faecal immunochemical test. United European Gastroenterol J. 2017;5(1): 134-138.

10. Hirata T, Kawakami Y, Kinjo N, et al. Association between colonic polyps and diverticular disease. World J Gastroenterol. 2008;14(15): 2411-2413.

11. Meurs-Szojda MM, Terhaar sive Droste JS, Kuik DJ, Mulder CJ, Felt-Bersma RJ. Diverticulosis and diverticulitis form no risk for polyps and colorectal neoplasia in 4,241 colonoscopies. Int J Colorectal Dis. 2008;23(10):979-984.

12. Peery AF, Martin CF, Levinson SE, Sandler RS. Colonic diverticula are not associated with an increased risk of colorectal adenomas. Am J Gastroenterol. 2015;110(12):1694-1697.

13. Nagata N, Niikura R, Aoki T, et al. Increase in colonic diverticulosis and diverticular hemorrhage in an aging society: lessons from a 9-year colonoscopic study of 28,192 patients in Japan. Int J Colorectal Dis. 2014;29(3):379-385.

14. Strate LL, Modi R, Cohen E, Spiegel BMR. Diverticular disease as a chronic illness: evolving epidemiologic and clinical insights. $\mathrm{Am} J$ Gastroenterol. 2012;107(10):1486-1493.

15. Yamada E, Inamori $\mathrm{M}$, Watanabe $\mathrm{S}$, et al. Constipation is not associated with colonic diverticula: a multicenter study in Japan. Neurogastroenterol Motil. 2015;27(3):333-338.

16. Lohsiriwat V, Suthikeeree W. Pattern and distribution of colonic diverticulosis: analysis of 2877 barium enemas in Thailand. World $J$ Gastroenterol. 2013;19(46):8709-8713.

17. Miura S, Kodaira S, Shatari T, Nishioka M, Hosoda Y, Hisa TK. Recent trends in diverticulosis of the right colon in Japan: retrospective review in a regional hospital. Dis Colon Rectum. 2000;43(10):1383-1389.

18. Cuomo R, Barbara G, Pace F, et al. Italian consensus conference for colonic diverticulosis and diverticular disease. United European Gastroenterol J. 2014;2(5):413-442.

19. Liu HH, Wu MC, Peng Y, Wu MS. Prevalence of advanced colonic polyps in asymptomatic Chinese. World J Gastroenterol. 2005;11(30): 4731-4734.

20. Ashktorab H, Panchal H, Shokrani B, et al. Association between diverticular disease and pre-neoplastic colorectal lesions in an Urban African-American Population. Digestion. 2015;92(2):60-65.

21. Fong SS, Tan EY, Foo A, Sim R, Cheong DM. The changing trend of diverticular disease in a developing nation. Colorectal Dis. 2011;13(3): 312-316.

22. Yamamichi N, Shimamoto T, Takahashi Y, et al. Trend and risk factors of diverticulosis in Japan: age, gender, and lifestyle/metabolic-related factors may cooperatively affect on the colorectal diverticula formation. PLoS One. 2015;10(4):e0123688.

23. Strate LL. Lifestyle factors and the course of diverticular disease. Dig Dis. 2012;30(1):35-45. 
24. Reichert MC, Lammert F. The genetic epidemiology of diverticulosis and diverticular disease: Emerging evidence. United European Gastroenterol J. 2015;3(5):409-418.

25. Burnett-Hartman AN, Passarelli MN, Adams SV, et al. Differences in epidemiologic risk factors for colorectal adenomas and serrated polyps by lesion severity and anatomical site. Am J Epidemiol. 2013;177(7): 625-637.

26. Tao S, Hoffmeister M, Brenner H. Development and validation of a scoring system to identify individuals at high risk for advanced colorectal neoplasms who should undergo colonoscopy screening. Clin Gastroenterol Hepatol. 2014;12(3):478-485.
27. Morrow EH. The evolution of sex differences in disease. Biol Sex Differ. 2015;6:5.

28. Bolnick DI, Snowberg LK, Hirsch PE, et al. Individual diet has sexdependent effects on vertebrate gut microbiota. Nat Commun. 2014;5: 4500.

29. He J, Gu D, Wu X, et al. Major causes of death among men and women in China. N Engl J Med. 2005;353(11):1124-1134.

\section{Publish your work in this journal}

Therapeutics and Clinical Risk Management is an international, peerreviewed journal of clinical therapeutics and risk management, focusing on concise rapid reporting of clinical studies in all therapeutic areas outcomes, safety, and programs for the effective, safe, and sustained use of medicines. This journal is indexed on PubMed Central, CAS,
EMBase, Scopus and the Elsevier Bibliographic databases. The manuscript management system is completely online and includes a very quick and fair peer-review system, which is all easy to use. Visit http://www.dovepress.com/testimonials.php to read real quotes from published authors.

Submit your manuscript here: http://www.dovepress.com/therapeutics-and-clinical-risk-management-journal 